Research Article

Kamal Barghout*

\title{
Analysis of repulsive central universal force field on solar and galactic dynamics
}

https://doi.org/10.1515/phys-2019-0041

Received Jun 30, 2018; accepted Apr 02, 2019

\begin{abstract}
Recent astrophysical observations hint toward the need for an extended theory of gravity to explain puzzles presented by the standard cosmological model such as the need for dark matter and dark energy to understand the dynamics of the cosmos. This paper investigates the effect of a repulsive central universal force field on the behavior of celestial objects. Negative tidal effect on the solar and galactic orbits, like that experienced by Pioneer spacecrafts, was derived from the central force and was shown to manifest itself as dark matter and dark energy. Vertical oscillation of the sun about the galactic plane was modeled as simple harmonic motion driven by the repulsive force. The proposed universal field was used to infer the shape of dark matter halos as generated from a planar component of the universal force and to explain galactic warp, galactic halo density, and galactic rotation curves. It was found that the repulsive field addition to Newton's gravity mimics the Yukawa potential correction employed by many current gravitational theories that modify gravity.
\end{abstract}

Keywords: Celestial mechanics, Dark matter, Pioneer anomaly, Simple harmonic motion, Yukawa potential

PACS: 95.30.-k, 98.10.+z, 95.35.+d, 95.36.+x

\section{Introduction}

The success of the standard cosmological model ( $\Lambda \mathrm{CDM})$ $[1,2]$ has been marred by a number of puzzling large scale cosmological observations $[3,4]$ which hint toward the need for modifications of the model. Therefore, extended theory of gravity may be needed since it is clear from recent astrophysical observations that Einstein equations are no longer good enough to test gravitation in the solar system and at the galactic scale, unless we admit some kind of ex-

*Corresponding Author: Kamal Barghout: Prince Mohammad Bin Fahd University, Alkhobar, Saudi Arabia;

Email: kbarghout@pmu.edu.sa

ə Open Access. ๑ 2019 K. Barghout, published by De Gruyter.

(cc) BY License otic matter-energy to the matter side of Einstein field equations, dubbed "dark matter" and "dark energy"; see [5] and references therein.

The existence of dark matter is mostly inferred from gravitational effects on visible matter and is thought to account for approximately $85 \%$ of the matter in the universe while dark energy is inferred from the accelerated expansion of the universe and along with dark matter constitutes about $95 \%$ of the total mass-energy content in the universe. The origin of dark matter is a mystery and a wide range of theories speculate its type, its particle's mass, its selfinteraction and its interaction with normal matter. Also, experiments to directly detect dark matter particles in the lab have failed to produce positive results which presents a serious challenge to its existence; see [6,7] and references therein. Nevertheless the hunt for a dark particle continues; such as the new approach to directly detect dark matter particles by observing them interacting with electrons from nuclear scattering [8]. Other problems that face the existence of dark matter is that simulations of dark matter according to current models that best describe it relevant to corresponding theories present further challenges when compared with observation; such as producing more steeply rising rotation curves than we see in many low surface brightness galaxies, suggesting that simulations produce an overabundance of dark matter [9] and when explaining the average velocity dispersions for the Milky Way and Andromeda, there should be five times more dark matter satellite galaxies according to theory [10]. For all of these challenges that face the proof of the existence of dark matter one may have to consider looking forward for alternate theories.

We propose that such as there has been equal amounts of normal baryonic matter and antimatter in the early universe with baryonic matter prevailing over antimatter due to matter-antimatter asymmetry, there has also been equal amounts of dark matter and anti-dark matter with dark matter prevailing and escaping complete destruction due to asymmetric anti-dark matter [11]. If that was the case, dark matter then might have separated and formed their own pockets in the universe. This scenario prompts the consideration of a long range gravitational interaction by 
dark matter "worlds" and our baryonic matter worlds. It is suggested here that this interaction might be negative and we can build on that to explain cosmological behavior of our baryonic worlds.

Attempts to explain various cosmological anomalies have not been very promising so far. To name few, attempts have been made to explain the Pioneer anomaly, ranging from systematic effects to new theoretical approaches [12$20]$, and to explain galactic rotation curves by invoking the existence of the perplexing dark matter [21-23] or by modifying the law of gravity [24, 25].

The concept of antimass was invoked by Albert Einstein himself. The newest in the literature in that regard is a "dark" fluid theory with repulsive behavior and constant creation of matter that help understand dark matter and dark energy at cosmological scales [26].

Classical theories of repulsive gravity have been published as extensions of general relativity where antiparticles "live" in an inverted spacetime [27-29]. Non-classical theories of repulsive gravity have also been published [30] Against general consensus in the field, the notion of antigravity that is based on the assumption that antimatter gravitationally repels matter drives scientists to test this possibility. Theories of that notion, if successful, must await confirmation by experiment, probably by ALPHA-g and GBAR experiments at CERN, which are yet to bear fruit as of current time.

In the weak-field limit of most of the current gravitational theories present today that try to explain the perplexing cosmological gravitational deviation from GR/Newtonian mechanics, such as nonsymmetric, scalartensor-vector (MOG), quantum gravitational theories of gravity...etc., result in a Yukawa-like type of gravitational potential [31-33]. For example, MOG is a covariant, relativistic, alternative gravitational theory whose field equations are derived from an action that supplements the spacetime metric tensor with vector and scalar fields. MOG predicts that far from the source of gravity, i.e., the core of a galaxy, the force field is stronger than the normal inverse-square Newtonian one, but at shorter distances, it is counteracted by a repulsive "fifth force" due to a vector field [34, 35]. MOG has been successfully applied to explain the rotation curves of the Milky Way galaxy [36] and the dynamics of galaxy clusters without dark matter. It has also been shown in a recent study that the theory satisfies the weak equivalence principle and is consistent with observations of the neutron star merger and gamma ray burster event GW170817/GRB170817A [37].

In a recent research, it was suggested that observational evidence for the existence of cold dark matter particles in the cores of dwarf galaxies could be explained through the interaction of a Yukawa potential [38]. Therefore it has been suggested that the experimental and observational search for an explanation of gravitational deviation from GR/Newton gravity might result in new type of physics [39]. Here, it is suggested that in a mass-antimass universe, the result of the existence of nearby aggregates of antimass could be a repulsive gravitational force field and would materialize as acceleration in the radial direction from the antimass aggregates, which presents a strong candidate to dark matter and dark energy, assuming antimass and normal mass gravitationally repel each other. In this scenario, the repulsive universal field serves as the source to the Yukawa field, or fifth force, that acts as a correction to the Newtonian classical potential in the mentioned gravitational theories.

In this paper, it is shown that galactic dynamics can be, in principle, achieved through extended theories of gravity. The existence of a force field originating from the center of the universe (assuming a spherical universe), possibly local universe, is proposed to drive much of the dynamics of the cosmos and explains many cosmic anomalies. This vector field could be the work of a constant repulsive force that could be the one responsible for the initiation of the Big Bang event and situated at the center of the universe or it could be the result of a nearby repulsive antimass aggregate. Here, the possibility of the existence of a universal repulsive force field is explored, and its effect on solar and galactic dynamics is studied since a model of extended gravity is pursued. To achieve that, a model of the universe with equal amounts of mass and antimass repelling each other and preserving a universal radial gravitational field is presented. Influenced by the repulsive universal force field, our Milky Way galaxy and its components feel the outward radial acceleration. A negative-tidal force resulting from a component of the repulsive central force acting on the solar system results in a sunward acceleration felt by the sun's orbiters. This is simply the opposite process of the tidal force that causes tides on earth. Here, Pioneer anomaly is explained mainly by considering the action of the negative central force field. It is demonstrated that the success of Newtonian mechanics in explaining the dynamics of the solar system can be extended to explain the rotational speed of stars in galaxies (galactic rotation curves) when corrected by the proposed repulsive universal force based on the assumption that Newtonian gravity is a good approximation without the need for large amounts of dark matter in galaxies. 


\section{Effect of a repulsive universal force on the solar system and galactic dynamics}

Consider a situation where the radial direction of the universe lies in the orbital plane of a planet in the solar system so the planet would be between the center of the universe and the sun at one point and on the opposite side after half orbital period. The proposed universal repulsive force will have a negative tidal effect on the solar system and can cause inward bulge (toward the sun) of the planet's orbit as acceleration of the sun in the upward direction is larger than that of the planet when the planet is situated above the sun relative to the center of the universe while the acceleration of the planet is larger than that of the sun when it is situated below the sun (or component of the repulsive force toward the sun when the orbital plane is tilted relative to the radial direction). Figure 1 shows the force acting on a celestial system, like our solar system, with an orbital plane perpendicular to the universal radial direction (tangential to a spherical shell concentric with the center of the universe).

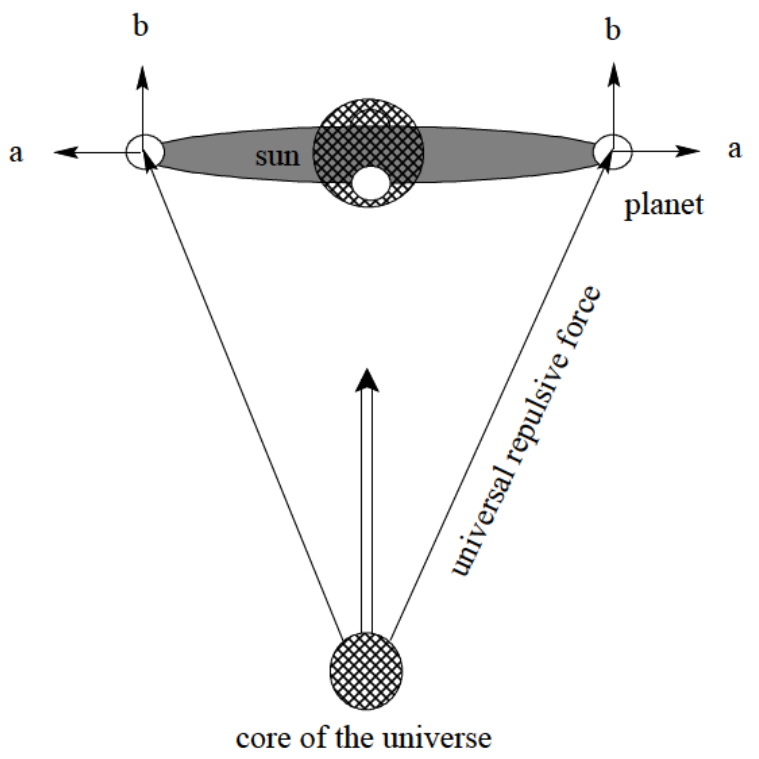

Figure 1: Universal repulsive force produces circular orbital shape of the planetary orbit in the absence of other effects when the orbit is perpendicular to the radial direction of the universal force

Similarly, the repulsive force affects the dynamics of galaxies. The unequal forces of $b_{1}$ and $b_{2}$ in an orbital plane of a star orbiting the galactic center that is not perpendicular to the universal radial direction as might be the case of the Milky Way imparts unequal accelerations relative to the position of the star with respect to the core of the universe. Repulsive tidal force also produces oscillation of the stars about their galactic plane as will be discussed in detail. While perpendicular components of the repulsive forces (b-forces in Figure 1) produce bulges of the orbital stars, the in-plane forces (a-forces in Figure 1) act as the restoring forces that produce oscillations of the stars about their galactic plane. Celestial systems then are oscillators oriented at random relative to each other due to the universal repulsive force acting on them.

The sun-orbiting objects, as in the case of the Pioneer spacecraft, feel negative tidal forces in all directions due to their orbital plane tilting relative to the tangential plane of the universal concentric shell at the location of the sun with varying magnitudes producing a bulge toward the sun as opposed to that of the normal positive tidal effect on earth by the sun and the moon.

\section{Pioneer anomaly}

Pioneer anomaly is a constant acceleration directed towards the Sun of

$a=(8.74 \pm 1.33) \times 10^{-10} \mathrm{~m} / \mathrm{s}^{2}$, registered by Pioneer spacecraft [40]. It is commonly believed that attempts to explain this anomaly gravitationally have failed so far.

Following the previous argument in section 2, the Pioneer Effect can be explained that due to the external universal repulsive force acting on the Pioneer spacecraft which produces negative tidal force that results in a constant sunward acceleration.

\section{Modeling the sun's dynamics as simple harmonic motion}

The sun oscillation about the galactic plane can be treated as simple harmonic motion modeled by a simple pendulum that extends from the center of the galaxy to the sun with the sun acting as the bob and a component of the repulsive central universal force (a-forces in Figure 1) as the restoring force. Using the period of the simple pendulum as the oscillation period of the sun to compute the gravitational field strength at the sun's location with the restoring forces pronounced when the galactic plane is perpendicular to the universal radial direction, the equation $P=2 \pi \sqrt{l / g}$; where the distance of the sun from the galaxy center is about 27700 ly and the oscillation period is about 
$66 \mathrm{M}$ years about the galactic plane, can be employed to give $\mathrm{g}$ (The gravitational field strength in the immediate neighborhood of the sun, which is assumed to originate from the repulsive force) as $0.24 \times 10^{-10} \mathrm{~m} / \mathrm{s}^{2}$. This should give a restoring force of the sun-galaxy pendulum of about $0.24 \times 10^{-10} \mathrm{M}_{\odot}$.

\section{Maximum height of stars from the galactic plane}

Models of gravitational force law perpendicular to the galactic plane allow the determination of the galactic velocity-distance relation with close fit to the velocity distance observations. Those models mainly assume an inplane galactic potential as entirely made by baryonic matter with observational confirmation of star counts, which increases the enigma of how a dark matter galactic halo is distributed in galaxies with the inner part of the galactic disk assumed to be mainly free of dark matter to explain perpendicular motion of stars about the galactic plane with unexplained in-plane dark matter-baryonic matter gravitational relationship. The perpendicular to the galactic plane velocity-distance relationship of stars obtained from the simple harmonic model of the sun agrees with observation and assumes the whole galaxy is free of dark matter as described in the introduction. Figure 2 illustrates the mechanism of the sun vertical oscillation about the galactic plane as modeled by simple harmonic motion.

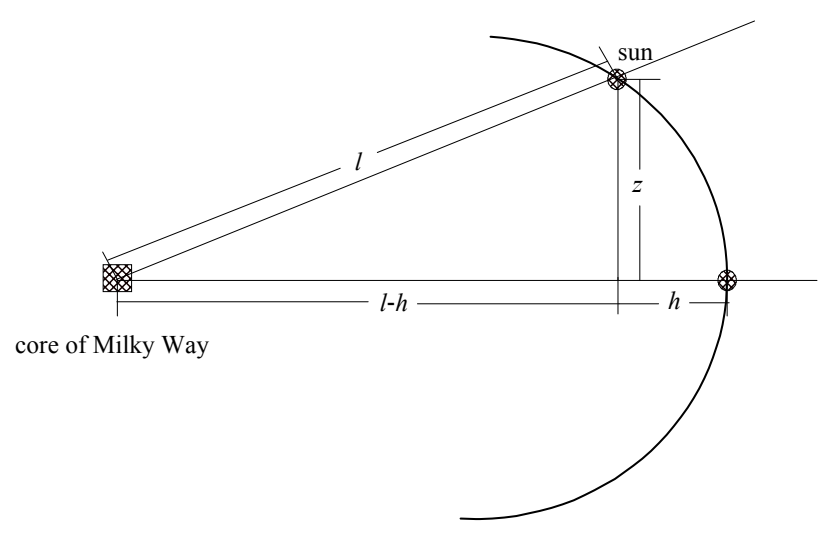

Figure 2: Vertical oscillation of the sun about galactic plane modeled as simple harmonic motion

As clear in Table 1, the sun galactic velocity-distance computed from the simple harmonic model is in close agreement with that from reference [41] computed from distribution function modeling technique.
Table 1: Sun galactic velocity-distance

\begin{tabular}{ccc}
\hline $\begin{array}{c}\text { Velocity } \\
\mathrm{m} / \mathrm{s} \text { at z=0 }\end{array}$ & $\begin{array}{c}\mathrm{Z}_{\text {maximum }}(\mathrm{Pc}), \\
\text { simple harmonic } \\
\text { model }\end{array}$ & $\begin{array}{c}\mathrm{Z}_{\text {maximum }}(\mathrm{Pc}), \\
\text { Kuikken \& Gilmore } \\
\text { paper }\end{array}$ \\
\hline 0 & 0 & 0 \\
8400 & 149 & 100 \\
15700 & 279 & 200 \\
21900 & 390 & 300 \\
27300 & 486 & 400 \\
32100 & 571 & 500 \\
36500 & 650 & 600 \\
40600 & 722 & 700 \\
44500 & 792 & 800 \\
48300 & 859 & 900 \\
51800 & 921 & 1000 \\
58600 & 1042 & 1200 \\
68300 & 1214 & 1500 \\
83200 & 1477 & 2000 \\
\hline
\end{tabular}

To compute the velocity-distance of the sun in Table 1 , the following governing equations have been used following the illustration in Figure 2.

$$
\begin{gathered}
v^{2}=2 g h \\
l^{2}=z^{2}+(l-h)^{2}
\end{gathered}
$$

where $g$ is the gravitational field strength at the sun vicinity, taken as $a=8.74 \times 10^{-10} \mathrm{~m} \mathrm{~s}^{-2}$, as that registered by Pioneer spacecraft, and $l$ is the distance of the sun-galaxy center taken as 27700 ly. Equation (1) is derived from conservation of mechanical energy with $v$ is the sun's velocity at $z=0$ and $h$ is the pendulum maximum height obtained from equation (1), obeying the equation $\frac{1}{2} m v^{2}=m g h$. Equation (2) is obtained from geometrical consideration as clear in Figure 2.

\subsection{Galactic disk warp}

Galactic disk warps can be explained in terms of the central universal repulsive force. At least half of all galaxies are warped and are long-lived or continuously generated. Galaxies with small dark halo core radii are less likely to be warped which indicates a direct relationship between galactic warp and dark matter halo. Also, it is thought that galactic warp is environmentally driven as galactic disks embedded in rotating halos experience a dynamical friction force which causes it to warp when the angular momentum axes of the disk and halo are misaligned [42]. 


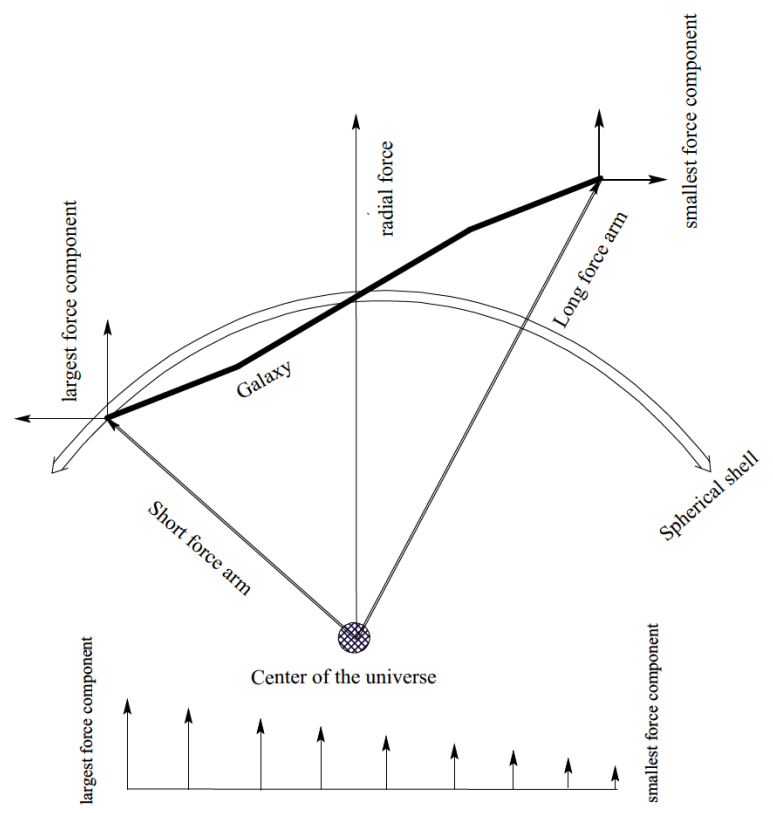

Figure 3: Galactic warp (not to scale)

Most of the galaxies with both sides warped are antisymmetric with S-shape warps. The warp of galaxies is normally explained in literature in terms of contained dark matter [43, 44]. In this paper dark matter is explained in terms of the central universal repulsive force and so is the galactic warp. Figure 3 shows the repulsive force acting on a proposed galaxy. If the galactic plane is not tangential to a spherical shell with its center as that of the universe, the central repulsive force will act with different magnitudes of perpendicular force components along the galactic plane. The longest arm of the force provides the smallest force component while the shortest provides the largest component because the whole galaxy is accelerated in the radial direction due to the central repulsive force but one end of the galaxy is accelerated faster than the opposite one, with perpendicular force distribution as shown in Figure 3. This produces warp of the galactic disk most pronounced away from the central bulge i.e. in a spiral galaxy. The warping mechanism made by the universal repulsive force clearly explains why the warps are S-shaped.

\section{Effect of repulsive force on the Milky Way galaxy at the sun vicinity (dark matter halo)}

We can approximate the "virtual" amount of DM at the sun's location due to the action of the proposed universal repulsive force. Since rotation curves of our galaxy show that the sun is moving about $60 \mathrm{~km} \mathrm{~s}^{-1}$ faster than what is contributed by visible matter, as the actual sun's velocity measures about $220 \mathrm{~km} \mathrm{~s}^{-1}$ while that contributed by visible matter is about $160 \mathrm{~km} \mathrm{~s}^{-1}$, the centripetal acceleration of a negative tidal force component on the sun can account for the difference with magnitude $0.24 \times 10^{-10} \mathrm{~m} \mathrm{~s}^{-2}$ (computed from the pendulum model); where $v=\sqrt{a l}$, $l$ is $27700 l y, v$ then is about $80 \mathrm{~km} \mathrm{~s}^{-1}$, in close agreement with observation. Accordingly, acceleration of about $0.24 \times 10^{-10} \mathrm{~m} \mathrm{~s}^{-2}$ may be used here to approximate the amount of DM enclosed by the sun's galactic orbit.

\section{Dark matter cosmic distribution}

It is easy to infer that the distribution of dark matter follows the distribution of baryonic matter by considering an external repulsive gravitational field as the cause of baryonic material behavior as discussed above to produce larger orbital velocities of galactic components than obtained by normal matter alone. This may account for what astrophysicists interpret as the work of an existing dark matter in the cosmos which only exists where baryonic matter exists.

\section{Halo density of galaxies}

It is known that LSB galaxies, mostly dwarf galaxies, have low luminosity and low baryonic mass and are much more dominated by dark matter than large galaxies [45]. As an example, our Milky Way is a large galaxy with about 50\% dark matter. Galaxies with $1 / 100$ of the luminosity of the Milky Way galaxy are about $90 \%$ dark. The smallest dwarf galaxies known are almost completely made of dark matter. In light of the universal repulsive force, this can be explained by that galactic dark matter content is a manifestation of the proposed universal repulsive force. Since dark matter halo is approximated by the formula $v=\sqrt{a l}$ and is dependent on the magnitude of the repulsive universal force, which in turn is dependent on the relative location of the host galaxy from the universal core, this results in that for the same size galaxies located at the same radial distance from the universal core, dark halos' content remains the same whereas baryonic mass density depends on baryonic content of the galaxies. Therefore, for dwarf galaxies of small baryonic content, dark matter halo density to baryonic density ratio is in principle larger. 


\section{Modeling repulsive mass-antimass universe and dark energy}

The universe can be modeled as one made with equal amounts of mass and antimass and of consecutive normal mass and antimass spherical shells of specified thickness with the outermost made from one kind considering that the repelling entity to normal mass is the antimass. The mechanism of the acceleration of the universe arises from the fact that matter of the outermost shell of the universe feels a net repulsive force due to the existence of net opposite mass bounded by the shell. This results in acceleration of the outer shell in the outward radial direction. The next inner shell (the second outermost shell) faces the same scenario and so on. An observer in any galaxy sees galaxies of his own shell accelerate away from each other with relative magnitudes since the whole shell increases in size by time except those galaxies that are aligned in the radial universal direction, which leads to that shells become thinner over time. This mechanism would be pronounced if our observable universe was only a patch of space in one shell. This accelerated movement of galaxies away from opposite kind galaxies and the self-aggregation of own kind galaxies leaves voids [46] among them which in principle accounts for the overall sponge-like structure of the universe. This is the same anywhere in the universe due to its spherical symmetry. The accelerated movement of galaxies in the context of the universal repulsive force presents strong candidate to "Dark Energy". Unlike matter creation theories, such as the "dark" fluid theory mentioned in the introduction, here, dark energy can be seen as the work of the universal repulsive force, hence there is no need for constant creation of matter in the cosmos as proposed by the dark fluid theory; see reference [26].

\section{Rotation curves and the shape of dark matter halo}

Observations of orbital velocities in spiral galaxies suggest a mass structure according to $v(r)=\left(r \frac{d \Phi}{d r}\right)^{\frac{1}{2}}$, where $\Phi$ is the galaxy's gravitational potential. Classical analysis of the orbital velocity of a star near the edge of a spiral galaxy that resides in the inner side of the outer most shell, as can be inferred from the proposed mass-antimass universe, is as follows: with equal amounts of mass and antimass in the universe, suppose that the last shell carries antimass and assuming symmetrical universe, this shell then encloses a net baryonic mass with the same magnitude. We can now apply Gaussian law to account for the gravitational field that pushes away a galaxy in the radial universal direction from the center of the concentric shells of galactic plane inclination angle $\theta$ relative to the universal radial direction. The governing equation is then $\int_{s} g . d a=4 \pi G m_{\text {enclosed }}$, where $m_{\text {enclosed }}$ is the shell's enclosed net baryonic mass. Considering the two-body system of the star-galaxy core, rotational speed of the star located near the edge of the galaxy can be approximated by,

$$
v=\left(\frac{G M_{\text {galaxy }}}{r}\right)^{\frac{1}{2}}+\left(\frac{G M^{\star}}{r}\right)^{\frac{1}{2}}
$$

where the second term serves as a correction to the Newtonian classical term and $M^{\star}$ is the "effective mass" due to $m_{\text {enclosed }}, r$ is the star-galactic center distance and the rotational speed component in the second term is a function of $\theta$. Note that the second term in equation (3) accounts for the negative tidal force acting on the star due to the existence of the universal repulsive force as explained earlier.

Rotation curves of galaxies (RCs) precisely describe their dynamics [47]. Since the distribution of stars in galaxies doesn't match observations of their rotation as expected from application of Kepler's laws, luminous matter alone does not account for the total mass of galaxies [48], prompting the existence of hidden dark matter.

RCs are used to describe dark matter distribution in halos [49]. It is well known that rotation curves of spiral galaxies are flat. This is usually attributed to the existence of halos surrounding galaxies that contain unseen dark matter, which should in theory constitute the missing mass.

Employing the proposed repulsive universal force field we can see in Figure 4 the universal force lines acting on a star with their relative angles.

We can see now how the universal repulsive force may account for the shape of the galactic rotation curves supposedly due to hypothetical dark matter: restricting the halo within the volume of the galaxy, we can infer from Figure 4 that the shape of the component of the galactic rotational speed of an edge star that lies on the galactic diameter of highest tilt (solid line in Figure 4), derived from the repulsive universal force, is governed by,

$$
v^{2}=\sin \alpha \cos (90-\theta-\alpha)
$$

To descriptively represent the shape of a galactic halo from the galactic center to the edge, equation (4) may be employed to approximate the rotational speed component of a star due to the repulsive universal force along the galactic diameter with maximum tilt. The formula was derived from the component of the centripetal acceleration 


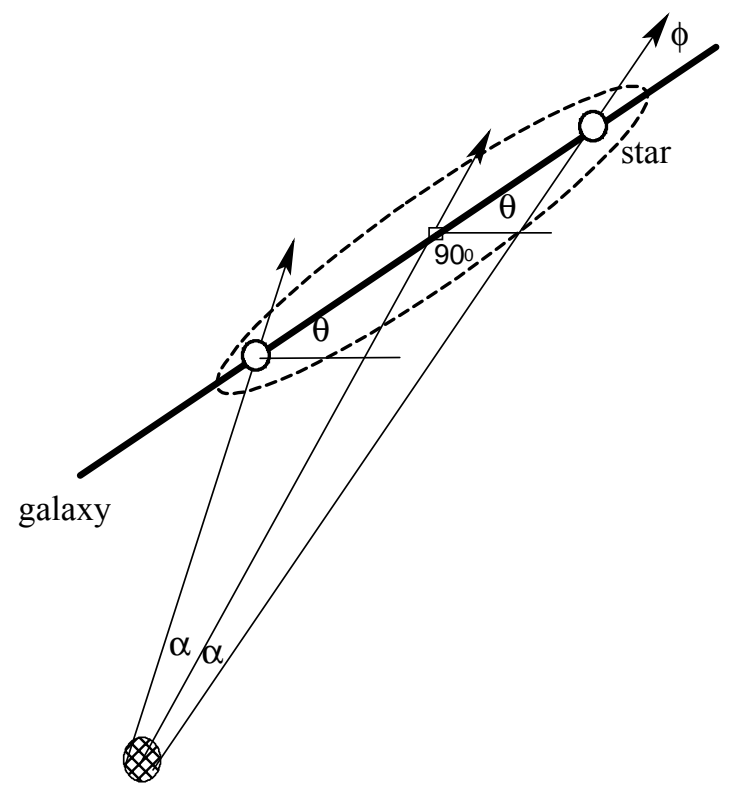

universal core

Figure 4: Universal repulsive force field lines on a star with their relative angles (not to scale)

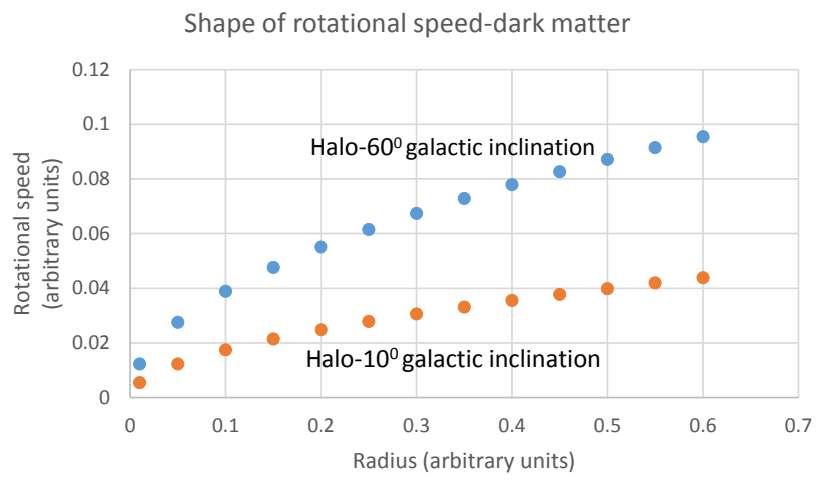

Figure 5: The shape of dark matter halo generated from galactic planar component of the universal repulsive force

due to the universal repulsive force of $v^{2} / d=\Phi \cos \varphi$, where $d$ is replaced by $\sin \alpha, \varphi=90-\theta-\alpha, \theta$ is the inclination angle of the galactic plane and $\Phi$ is the gravitational field due to the universal repulsive force and approximated to have the same value everywhere at the location of the galaxy with a value of unity. In Figure 5, the shape of the halo is inferred by plotting the rotational speed of the star against the angle $\alpha$ representing the galactic radius.

As expected, the increase of the inclination angle increases dark matter halo density as shown in Figure 5. Also, the halo density increases with radius which produces flattening of the RCs as opposed to Keplerian rotation.

\section{Repulsive universal force as the source of a Yukawa-like field}

Current research on gravitational theories explores nonNewtonian component of the gravitational field and presents it as a Yukawa-type correction to the classical gravitational potential and successfully describes various observational astrophysical and cosmological phenomena without resorting to dark matter; see references [31-33]. For a two-body system of a secondary body of mass $m$ under the gravitational influence of a primary body of mass $M_{p}$, the secondary body's potential energy can be described by additional modified term [50] as follows,

$$
V(r)=-\frac{G M_{p} m}{r}\left(1+\alpha e^{-\frac{r}{\lambda}}\right)=V_{N}(r)+V_{Y K}(r)
$$

where $G$ is the gravitation constant, $\alpha$ is the strength of the Yukawa interaction relative to gravity, $\lambda$ is its range, and $r$ is the separation distance between the two bodies. The Yukawa modified Newtonian potential is used to study a wide range of cosmological gravitational phenomena that pertain to deviation from normal celestial kinematics and even to estimate the mass of the graviton mediating the interaction in celestial bodies; see reference [32]. Similarly, the modified gravity (MOG) theory introduces a Yukawa-like modification term to Newton's gravity; see reference [37] and references therein. Among extra dimension theories, models with large extra dimensions propose that the interaction between massive objects by exchanging light KK graviton successfully modify Newton's law of universal gravitation at small distances by adding a Yukawa potential to the ordinary Newtonian potential in the static limit [51].

The gravitational correction to Newton's law on a twobody satellite system as described by equation (3) mimics the Yukawa potential correction. The second term in the equation replaces the Yukawa correction term and introduces a source of the potential as originating from the proposed negative central mass in a mass-antimass universe that produces a tidal force on celestial objects as described in this paper, where the effective mass " $M^{\star}$ " is interpreted as the mass contribution by the source that produces the Yukawa-like field. Since $M^{\star}$ depends on the location of the two-body system relative to the repulsive source (center of the universe), and thereby the value of the potential $\Phi$ in the equation $v^{2} / d=\Phi \cos \varphi$, we conclude that the rest of the parameters involved in equations (3) and (4) must be observation-dependent. Observational verification to the gravitational correction by the universal gravitational field is presented through studying the kinematics of the sun in 
sections 4, 5 and 7. A quantitative assessment of a universal repulsive field acting on a celestial object, perhaps a two-body celestial system, is intended for future work by parameterizing equations (3) and (4) and fitting available cosmological data.

\section{Conclusion}

As an extended gravity model, it was shown that by accounting for repulsive component of a universal force field in Newtonian mechanics we can provide reasonable explanation to several cosmic puzzles such as dark matter, dark energy, rotation curves, Pioneer anomaly, and galactic disk warp. Importantly, it was shown that negative tidal effect on solar orbits may explain the extra sunward acceleration experienced by Pioneer spacecrafts. The negative tidal force was used to show similar inward acceleration experienced by stars in their galactic orbits and descriptively was used to show how it manifests itself to produce non-Keplerian rotation curves. Similarly, the shape of dark matter halo was inferred by employing the negative tidal force. Vertical oscillation of the sun about the galactic plane was modeled as simple harmonic motion driven by a component of the universal repulsive field and was compared with another model computed from distribution function modeling technique. Accordingly, it was found that the repulsive component of the proposed universal field mimics a Yukawa potential correction to the normal Newtonian field which recently has attracted much attention in the current gravitational theories that modify gravity.

Acknowledgement: I would like to acknowledge the encouragement and support by Prince Mohammad Bin Fahd University. I also would like to express my appreciation to the reviewers whose inputs were invaluable addition to the manuscript.

\section{References}

[1] Perivolaropoulos L., Large scale cosmological anomalies and inhomogeneous dark energy, Galaxies, 2014, 2(1), 22-61.

[2] Hamilton J.-C., What have we learned from observational cosmology? 2013, arXiv:1304.4446.

[3] Perivolaropoulos, L., Six puzzles for LCDM cosmology, ArXiv EPrints, 2008, arXiv:0811.4684.

[4] Yang, R.J., and Zhang, S.N., The age problem in $\Lambda$ CDM model, Mon. Not. R. Astron. Soc., 2010, 407, 1835-1841.
[5] Corda, C., Interferometric Detection of Gravitational Waves: The Definitive Test for General Relativity. International Journal of Modern Physics D, 2009, 18, 2275-2282.

[6] Arun K., Gudennavar S. B., and Sivaram C., Dark matter, dark energy, and alternate models: A review, ArXiv E-Prints, 2017, arXiv: 1704.06155.

[7] Garrett K., and Duda G., Dark matter: A primer, Advances in Astronomy, 2010, 2011(6), 968283.

[8] Dolan M. J., Kahlhoefer F., and McCabe C., Directly detecting sub$\mathrm{GeV}$ dark matter with electrons from nuclear scattering, Phys. Rev. Lett., 2018, 121, 101801.

[9] Klypin A., Kravtsov A. V., Valenzuela O., and Prada F., Where are the missing galactic satellites? Astrophysical Journal, 1999, 522(1), 82-92.

[10] Moore B., Quinn T., Governato F., Stadel J., and Lake G., Cold collapse and the core catastrophe, Monthly Notices of the Royal Astronomical Society, 1999, 310(4), 1147-1152.

[11] Frandsen M. T., and Sarkar S., Asymmetric dark matter and the Sun, Phys. Rev. Lett., 2010, 105, 011301.

[12] Anderson J. D., Laing P. A., Lau E. L., Liu A. S., Nieto M. M., and Turyshev S. G., Indication, from Pioneer 10/11, Galileo, and Ulysses data, of an apparent anomalous, weal, long-range acceleration, Phys. Rev. Lett., 1998, 81, 2858.

[13] Turyshev S. G., Toth V. T., Kellogg L.R., Lau E. L., and Lee K. J., A study of the Pioneer anamoly: new data and objectives for new investigation, International Journal of Modern Physics, 2006, 15, 01, 1-55.

[14] Bertolami O., and Paramos J., The Pioneer anomaly in the context of the braneworld scenario, Class. Quantum Grav., 2004, 21 3309.

[15] Turyshev S. G., Toth V. T., Kinsella G., Lee S.-C., Lok S. M., Ellis J., Support for the thermal origin of Pioneer anomaly, Physical Review Letters, 2012, 108 (24).

[16] Anderson J.D., Lau E. L., Turyshev S.G., Laing P.A., and Nieto M.M., Search for the standard explanation of Pioneer anomaly, Mod. Phys. Lett., 2002, A17, 875.

[17] Jaekel M.T., and Reynaud S., Gravity tests in the solar system and Pioneer anomaly, Mod. Phys. Lett., 2005, A20 1047.

[18] Ranada A.F., The Pioneer anomaly as acceleration of the clocks, Found. Phys., 2005, 341955.

[19] Nottale L., The Pioneer anomalous acceleration: a measurement of the cosmological constant at the scale of the solar system, ArXiv E-Prints, 2011, arXiv: gr-qc/0307042.

[20] Foot R., and Volkas R.R., A mirror world explanation for the Pioneer spacecraft anomalies? Phys. Lett., 2001, B 51713.

[21] Weinberg D. H., Colombi S., Dave R., and Katz N., Baryon dynamics, dark matter substructure, and galaxies, The Astrophysical Journal, 2008, 678 (1): 6-21.

[22] Duffy A. R., Schaye J., Kay S. T., Vecchia C. D., Battye R. A., and Booth C. M., Impact of Baryon physics on dark matter structure: a detailed simulation study of halo density profiles, Monthly Notices of the Royal Astronomical Society, 2010, 405(4):21612178.

[23] De Blok W. J. G., and McGaugh S., The dark and visible matter content of low surface brightness disc galaxies, Monthly Notices of the Royal Astronomical Society, 1997, 290 (3): 533-552.

[24] Milgrom M., A modification of the Newtonian dynamics as a possible alternative to hidden mass hypothesis, Astrophysical Journal, 1983, 270: 365-370. 
[25] Milgrom M., MOND Laws of Galaxy Dynamics, ArXiv E-Prints, 2013, arXiv: 1212.2568 .

[26] Farnes J.S., A unifying energy of dark energy and dark matter: negative matter and mass creation within a modified $\Lambda$ CDM framework, Astronomy \& Astrophysics, 2018, 620: A92.

[27] Santilli R.M., A classical isodual theory of antimatter and its prediction of antigravity, International Journal of Modern Physics A, 1999, 14 (14): 2205-2238.

[28] Villata M., CPT symmetry and antimatter gravity in general relativity, EPL., 2011, 94 (2): 20001.

[29] Villata M., On the nature of dark energy: the lattice universe, Astrophysics and Space Science, 2013, 345 (1): 1-9.

[30] Cabbolet M. J. T. F., Addendum to the elementary process theory, Annalen der Physik, 2011, 523 (12): 990-994.

[31] Piazza F., and Marinoni C., Models for Gravitational Interaction between Dark Matter and Baryons, Phys Rev Lett., 2003, 91, 14.

[32] Martz C., Middelkoop S. V., Gkigkitzis I., Haranas I., and Kotsireas I., Yukawa potential orbital energy: it relation to orbital mean motion as well to graviton mediating the interaction in celestial bodies, Advances in Mathematical Physics, 2019, 2019, 6765827.

[33] Mukherjee R., and Sounda S., Single particle closed orbits in Yukawa potential, Indian Journal of Physics, 2018, 92 (2), 197203.

[34] Moffat J. W., Scalar tensor vector gravity theory, Journal of Cosmology and Astroparticle Physics, 2006, 3 (3): 4.

[35] Moffat J. W., and Toth V. T., Testing modified gravity with globular cluster velocity dispersions, Astrophysical Journal, 2008, 680 (2): 1158-1161.

[36] Negrelli S. C., Benito M., Landau S., locco F., and Kraiselburd L., Testing modified gravity theory in the Milky Way, Phys. Rev. 2018, D 98, 104061.

[37] Green M. A., Moffat J. W., and Toth V. T., Modified gravity (MOG), the speed of gravitational radiation and the event GW170817/GRB170817A, Physics Letters B, 2017, 780, 300-302.

[38] Chan H. M., Observational evidence of the Yukawa potential interacting dark matter, The Astrophysical Journal Letters, 2013, 91, 14, 20032.
[39] Adelberger E. G., Heckel B. R., and Nelson A. E., Test of the gravitational inverse square law, Annual Review of Nuclear and Particle Science, 2003, 53, 77-121.

[40] Anderson J. D., Laing P. A., Lau E.L., Liu A. S., Nieto M. M., and Turyshev S. G., Indication from Pioneer $10 / 11$ Galileo, and Ulysses data, of an apparent anomalous, weak, long-range, acceleration, Phys. Rev. Lett., 1998, 81, 2858.

[41] Kuiken K., and Gilmore G. F., The mass distribution in galactic disc -II- determination of the surface mass density of the galactic disc near the sun, MNRAS, 1989, 239, 605.

[42] Debattista V.P., and Sellwood J.A., Warped galaxies from misaligned angular momenta, The Astrophysical Journal, 1999, 513:L107 - L110.

[43] Sparke L.S., and Casertano S., A model for persistent galactic warps, MNRAS, 1988, 234, 873.

[44] Binney J., Warps, ARA\&A, 1992, 30, 51.

[45] Se-Heon O., Deidre A. H., Elias B., Bruce G. E., Andreas S., Fabian W., et al., High-resolutions mass models of dwarf galaxies from LITTLE THINGS, The Astronomical Journal, 2015, 149, 6.

[46] Nella H. Di, Couch W., Parker Q., and Paturel G., Unveiling a connection between large-scale structures behind the southern Milky Way, MNRAS, 1997, 287, 472.

[47] Sofue Y., and Rubin V. C., Rotation curves of spiral galaxies, ARAA, 2001, 39, 137.

[48] Rubin V., Thonnard N., Ford W. K. Jr., Rotational properties of 21 $\mathrm{SC}$ galaxies with a large range of luminosities and radii, from NGC $4605 / R=4 \mathrm{kpc} /$ to UGC $2885 / R=122 \mathrm{kpc} /$, The Astrophysical Journal, 1980, 238: 471.

[49] Honma M., and Sofue Y., Rotation curve of the galaxy, PASJ, 1997, 49, 453.

[50] Fischbach E., Talmadge C., and Krause D.E., Exponential models of non-Newtonian gravity, Phys. Rev. D, 1991, 43, 460.

[51] Schmitz K., Experimental Tests of the Gravity Sector, DESY Werkstatt seminar, Physics of Extra Dimensions, 2011, Summer Term. 